\title{
Photoacoustic microscopy of complex regional pain syndrome type I (CRPS-1) after stellate ganglion blocks in vivo
}

Yong Zhou, Xiaobin Yi, Wenxin Xing, Song $\mathrm{Hu}$, Konstantin I. Maslov, et al.

Yong Zhou, Xiaobin Yi, Wenxin Xing, Song Hu, Konstantin I. Maslov, Lihong V. Wang, "Photoacoustic microscopy of complex regional pain syndrome type I (CRPS-1) after stellate ganglion blocks in vivo," Proc. SPIE 9323, Photons Plus Ultrasound: Imaging and Sensing 2015, 932338 (11 March 2015); doi: $10.1117 / 12.2076691$ 


\title{
Photoacoustic microscopy of complex regional pain syndrome type I (CRPS-1) after stellate ganglion blocks in vivo
}

\author{
Yong Zhou, ${ }^{1}$ Xiaobin Yi ${ }^{2}$ Wenxin Xing, ${ }^{1}$ Song Hu, ${ }^{1}$ Konstantin I. Maslov, ${ }^{1}$ and Lihong V. \\ Wang ${ }^{1}$ \\ ${ }^{1}$ Washington University in St. Louis, Department of Biomedical Engineering, Optical Imaging \\ $2 \quad$ Laboratory, One Brookings Drive, Campus Box 1097, St. Louis, MO 63130 \\ ${ }^{2}$ Washington University School of Medicine, Department of Anesthesiology/Pain Management, \\ 660 South Euclid Avenue, Campus Box 8054, St. Louis, MO 63110 \\ *Correspondence: Xiaobin Yi, (pain studies), Email: yix@anest.wustl.edu; Lihong V. Wang, \\ (photoacoustic imaging), Email: 1hwang@seas.wustl.edu
}

\begin{abstract}
We used photoacoustic microscopy (PAM) to assist diagnoses and monitor the progress and treatment outcome of complex regional pain syndrome type 1 (CRPS-1). Blood vasculature and oxygen saturation $\left(\mathrm{sO}_{2}\right)$ were imaged by PAM in eight adult patients with CRPS-1. Patients' hands and cuticles were imaged both before and after stellate ganglion block (SGB) for comparison. For all patients, both the vascular structure and $\mathrm{sO}_{2}$ could be assessed by PAM. In addition, more vessels and stronger signals were observed after SGB.

Keywords: Complex regional pain syndrome, photoacoustic microscopy, stellate ganglion block
\end{abstract}

\section{Introduction}

Complex regional pain syndrome (CRPS) is a pain disorder characterized by severe and disproportionate pain, autonomic changes such as abnormal regulation of blood flow and sweating, edema of skin and subcutaneous tissues, motor disorders, and dystrophic changes. The pathophysiological mechanism of CRPS is still poorly understood, although our knowledge of this syndrome has increased significantly [1-3]. Multiple factors, including vascular and microcirculatory dysfunction, neurogenic inflammation [4], alteration in sympathetic and catecholaminergic function, as well as peripheral and central sensitization, may all play important roles in the pathogenesis of this disabling syndrome [5]. However, microcirculatory parameters, including rate and volume of blood flow and tissue oxygen saturation $\left(\mathrm{sO}_{2}\right)$, are of considerable interest for potentially diagnosing CRPS and monitoring its progress, due to the connection between the microvascular blood supply and the development of neuropathic pain [6].

There is increasing evidence to show that inflammatory processes and immune reactions are involved in the pathophysiology of CRPS [7]. In a systemic review and meta-analysis study, Parkitny et al [4] concluded that CRPS is associated with proinflammatory states in the blood, blister fluids, and cerebrospinal fluid (CSF). The CRPSrelated inflammation may change the sympathetic tone of blood vessels and therefore affect blood supply and tissue oxygenation. The acute and chronic phases of CRPS demonstrate different inflammatory features in both clinical manifestations and inflammatory profiles. The measurement of microcirculatory parameters, such as blood flow rate, blood volume, and blood oxygen saturation $\left(\mathrm{sO}_{2}\right)$ can be used to potentially diagnose the presence of CRPS, to indicate the activity of the disease, and to monitor the effectiveness of the therapeutic intervention.

In pain studies, traditional techniques for detecting microcirculatory parameters include functional magnetic resonance imaging (fMRI) and positron emission tomography (PET)[8]. However, both have limitations: fMRI makes indirect measurements by the blood-oxygenation-level dependent (BOLD) method [9, 10] or makes direct flow measurements with low sensitivity by the arterial spin labeling (ASL) method [11]; PET has to use radioactive tracers to measure blood flow directly [12]. To overcome these limitations, optical methods provide alternative ways to make direct, sensitive, non-invasive, and non-ionizing measurements of blood flow and $\mathrm{sO}_{2}$ in pain studies [1316]. Optical spectroscopic methods, such as near-infrared spectroscopy (NIRS) [13] and micro-lightguide

Photons Plus Ultrasound: Imaging and Sensing 2015, edited by Alexander A. Oraevsky, Lihong V. Wang

Proc. of SPIE Vol. 9323, 932338 - ( 2015 SPIE · CCC code: $1605-7422 / 15 / \$ 18$ doi: $10.1117 / 12.2076691$

Proc. of SPIE Vol. 9323 932338-1 
spectrophotometry [14], can provide non-invasive measurement of average blood flow and $\mathrm{sO}_{2}$, but with poor spatial resolution. Optical coherence tomography and optical Doppler imaging $[15,16]$ provide blood vasculature anatomy imaging with high spatial resolution and sensitive measurements of flow speed, but lack high sensitivity for measuring $\mathrm{sO}_{2}$, because their imaging contrasts are based on optical scattering.

Recently, photoacoustic microscopy (PAM) has emerged as a powerful modality for biomedical imaging [17-19]. In PAM, tissue is irradiated by a short-pulsed laser beam. Some of the light is absorbed and partially converted to heat, which subsequently induces acoustic waves (termed photoacoustic (PA) waves) via thermoelastic expansion. Because hemoglobin has strong absorption in the visible spectral region, PAM has high sensitivity in blood vasculature anatomy imaging [20] and flow imaging [21, 22]. In addition, because oxygenated hemoglobin $\left(\mathrm{HbO}_{2}\right)$ and deoxygenated hemoglobin $(\mathrm{Hb})$ have different absorption spectra, $\mathrm{PAM}$ can also accurately measure $\mathrm{sO}_{2}$. Thus, all the aforementioned microcirculatory parameters including blood flow rate, blood volume, and blood oxygen saturation $\left(\mathrm{sO}_{2}\right)$ can be measured by PAM. So far, functional PAM has been successfully demonstrated in both animals [23-25] and humans [26].

The diagnosis of CRPS is based on clinical manifestations, and there are limited laboratory tests or image studies to verify the diagnosis. With its high spatial resolution and label-free nature, PAM is potentially an ideal tool for monitoring and diagnosing CRPS by imaging peripheral blood perfusion. In this work, we performed a series of experiments to show the feasibility of this new imaging tool.

(a)

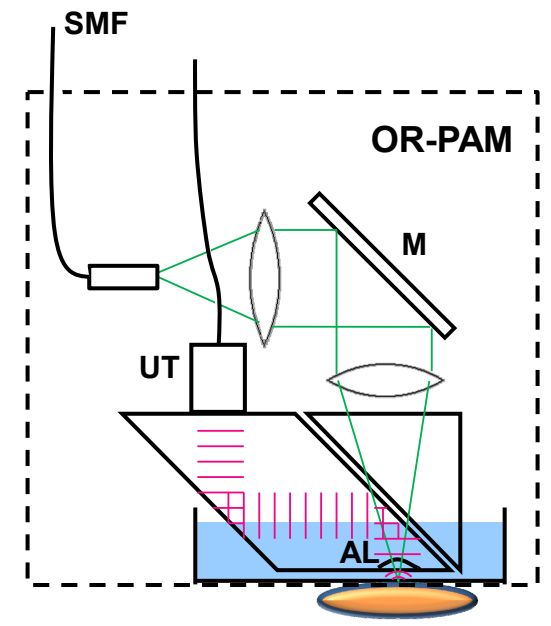

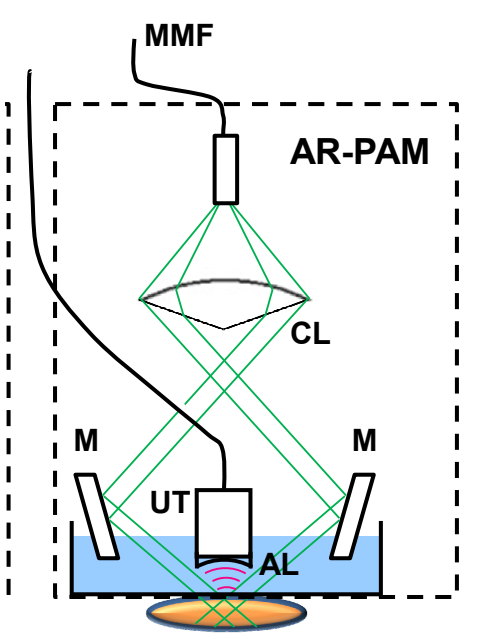

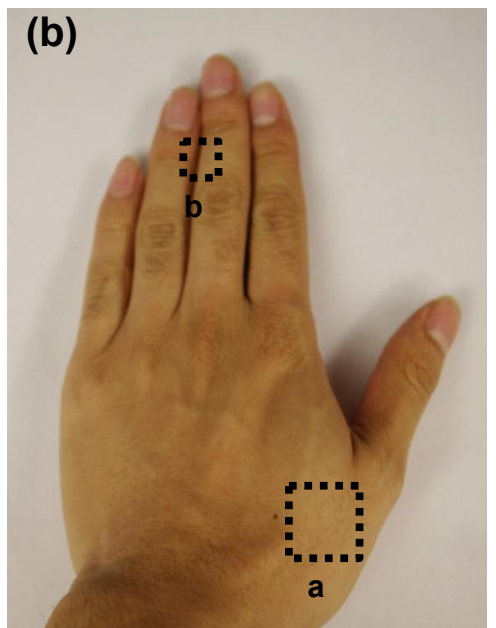

Fig. 1. (a) Schematic of the OR-PAM and AR-PAM systems. AL: acoustic lens; CL: conical lens; M: mirror; MMF: multi-mode fiber; SMF, single mode fiber; UT: ultrasound transducer. (b) Photograph of the hand, showing the scanned regions. a: region scanned by AR-PAM; b: region scanned by OR-PAM.

\section{Methods}

In this prospective observational study, we screened patients with a possible diagnosis of CRPS -1 who were referred to the Pain Management Center of Washington University in St. Louis between January 2011 and December 2011. Nine adult patients (28-69 years old, 6 female, 3 male) with CRPS-1 were recruited. Patients with pain in only one upper extremity were selected to participate in the study. One patient withdrew from the study. Patients were considered to be candidates for the study if they were diagnosed with CRPS-1 by using the proposed new diagnostic criteria of the International Association for the Study of Pain (IASP) [27]. All patients signed consents to be involved in the study. All human research procedures were carried out in conformity with the protocol approved by the Institutional Review Board at Washington University in St. Louis. For all the patients, symptoms and signs of CRPS were present for less than one year. Average pain level was 5 or higher on a numerical rating score (NRS) scale of $0-10$ (with 0 being no pain and 10 being the worst imaginable pain). Exclusion criteria 
were pregnancy, coagulopathy, fever, general infection or local infection at the block puncture site, substance abuse, peripheral neuropathy or any other diseases that may account for signs and symptoms mimicking CRPS. The patient was observed in the recovery area for 15 mins after the procedure. 10 mins after SGB, skin temperature in the block side increased $1.1 \pm 0.1{ }^{\circ} \mathrm{C}$. Meanwhile, pain level reduced by $2.5 \pm 0.6$ on discharge.

The experimental paradigm consisted of two imaging sessions, with a SGB between the sessions. For each patient, the back of their hand and the cuticle of the same hand were imaged before SGB (baseline) and then after SGB. Two sites (Fig. 1b) were chosen to image: superficial microvasculature at the cuticle and deeper and larger vessels at the back of the hand.

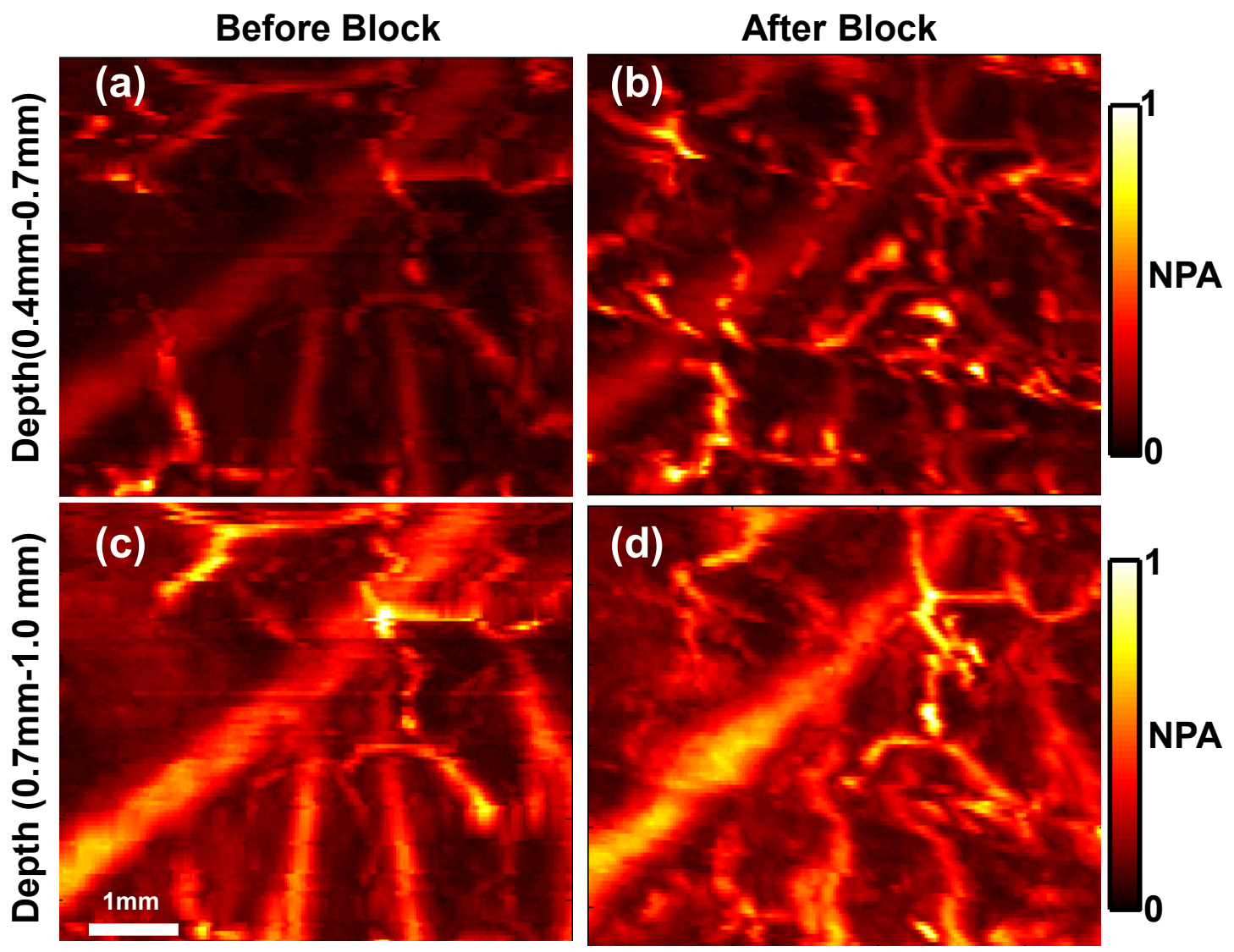

Fig. 2. MAP (maximum amplitude projection) image of vasculature in the back of one patient's hand by AR-PAM. MAP image in the depth range between $0.4 \mathrm{~mm}$ and $0.7 \mathrm{~mm}$ before block (a) and after block (b). MAP image in the depth range between $0.7 \mathrm{~mm}$ and $1 \mathrm{~mm}$ before block (c) and after block (d). NPA: Normalized photoacoustic amplitude.

According to its light illumination mechanism, PAM can be classified into two categories: acoustic-resolution (AR-) PAM and optical-resolution (OR-) PAM. In AR-PAM, the light is weakly focused, and the lateral resolution is determined by the acoustic focusing of the ultrasonic transducer. In OR-PAM, the light is tightly focused, and thus the lateral resolution is determined by the optical focusing provided by the objective. The axial resolution of both AR- and OR-PAM is determined by the bandwidth of the transducer used in the system. Usually optical focusing is much tighter than acoustic focusing, and thus OR-PAM has a better lateral resolution than AR-PAM. However, since acoustic scattering is much weaker than optical scattering in soft tissue, AR-PAM can focus deeper than ORPAM. To monitor hemodynamic changes with both high spatial resolution and deep penetration, both AR- and ORPAM systems were used in this research. The schematics are shown in Fig. 1(a). In the AR-PAM system, a donutshaped illumination is formed when light passes through the conical lens. A 20-MHz ultrasonic transducer collects the PA signals. In the OR-PAM system, light illumination and acoustic collection are achieved by an ultrasoundoptical beam combiner. A 50-MHz ultrasonic transducer detects the PA signals. Each laser pulse generates a one- 
dimensional depth-resolved image ("A-line") in both the AR- and OR-PAM systems. By two-dimensional rasterscanning of the entire area, a three-dimensional image can be acquired. For more accurate $\mathrm{sO}_{2}$ calculation in both AR- and OR-PAM systems, a photodiode compensates for the local fluence fluctuation. A detailed system description can be found in our previous papers [28-31]. The lateral resolutions of the AR- and OR-PAM systems are $80 \mu \mathrm{m}$ and $3 \mu \mathrm{m}$, respectively. To measure $\mathrm{sO}_{2}$, light at two wavelengths, $570 \mathrm{~nm}$ and $578 \mathrm{~nm}$, was used in both AR- and OR-PAM.

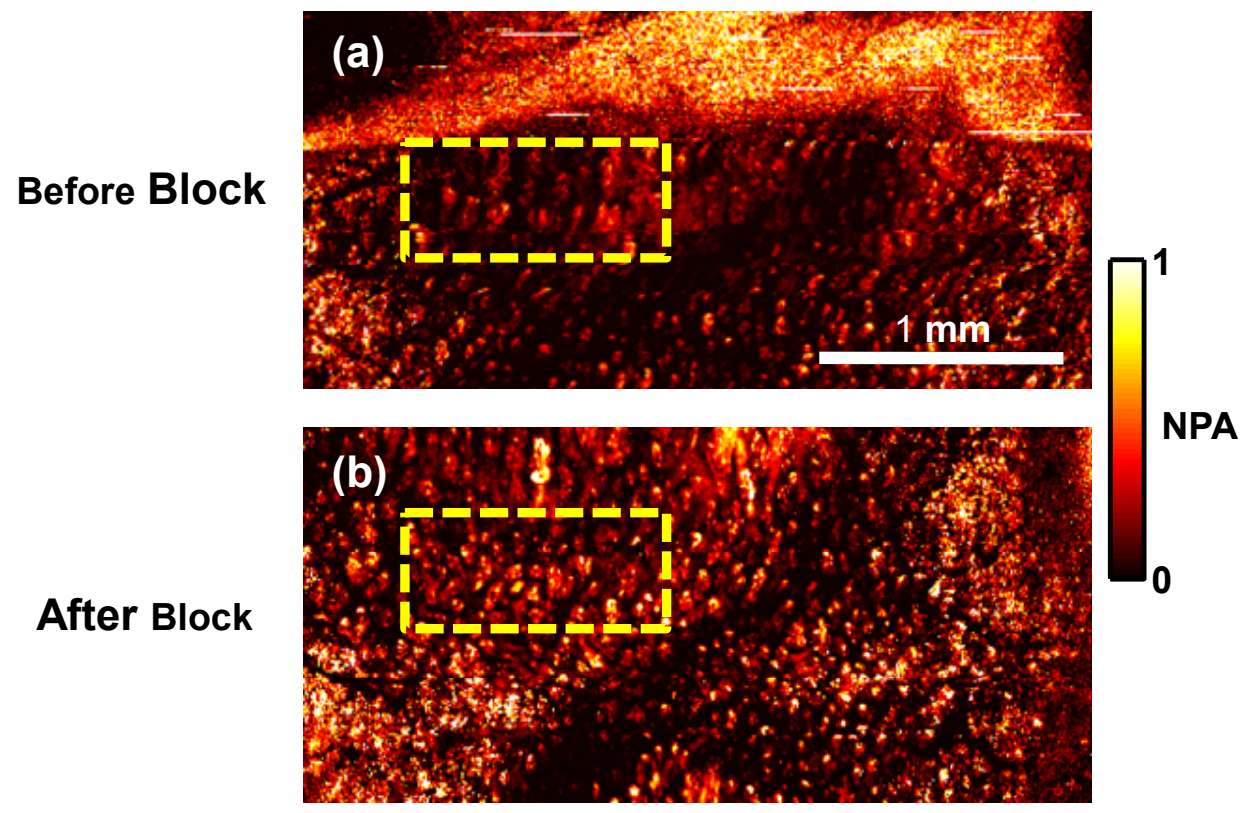

Fig. 3. Vasculature image in the finger tip of one patient by OR-PAM. MAP of scanned region before block (a) and after block (b). The dashed-box-represents the region used for statistical analysis.

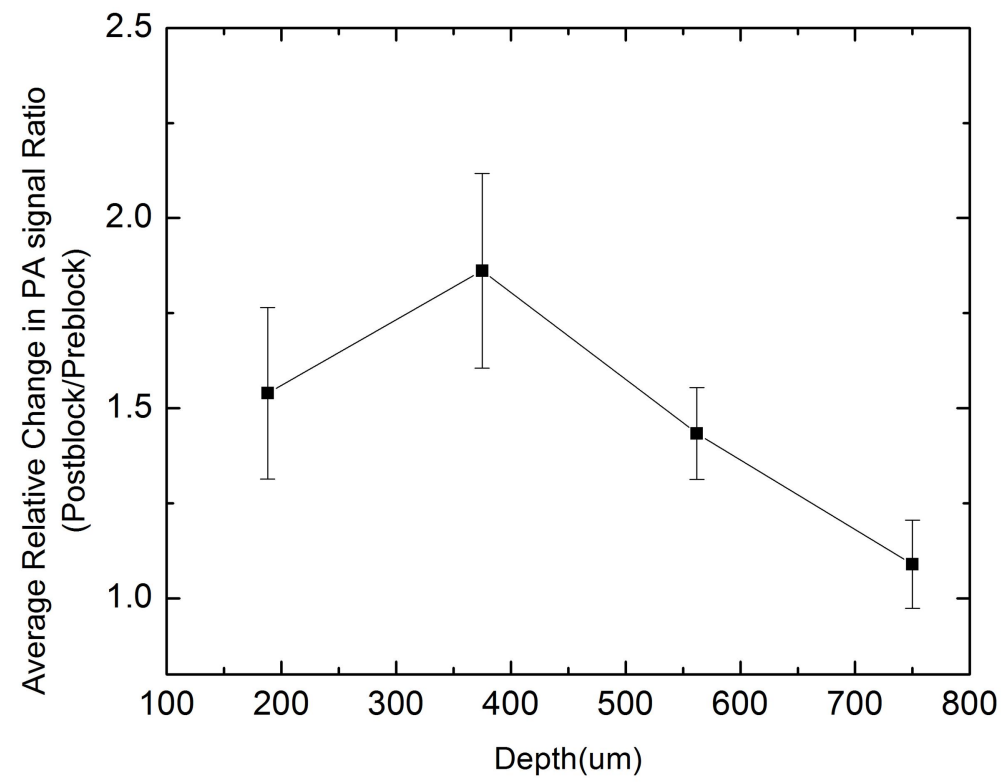

Fig. 4. Normalized PA signal measured by AR-PAM before and after block in different depth. 


\section{Results}

Typical AR- and OR-PAM hand images of patients are shown in Figs. 2 and 3, respectively. For fair comparisons, the distance between the patient's hand and the ultrasonic transducer was adjusted to be the same before and after SGB for imaging. In AR-PAM, a $\sim 6 \mathrm{~mm}$ by $6 \mathrm{~mm}$ field of view was scanned. The signals down to $1 \mathrm{~mm}$ deep were collected. Due to the high axial resolution of AR-PAM, we were able to differentiate signals from different depths. Maximum-amplitude-projection (MAP) images along the depth direction, with ranges of $0.4 \mathrm{~mm}-0.7 \mathrm{~mm}$ and 0.7 mm-1.0 mm, are shown in Fig. 2. Two major differences can be easily seen: more blood vessels and stronger signals after the block. Because the local optical fluence fluctuation had been compensated for, the PA signal was mainly dependent on the absorption coefficient of the target. Thus, an increase in the PA signal indicates an increase in the local absorption coefficient, probably due to increased blood concentration or volume. Therefore, we suspect that there was increasing blood perfusion and blood vessel dilation after the block. Typical blood vessels in the finger cuticle imaged by OR-PAM are shown in Fig. 3. As shown in Fig. 3, a stronger signal was found after the block, which is consistent with the results from AR-PAM.

Statistical analysis of all the patient data are shown in Fig. 4. Fig. 4 shows the average increase in signal intensity at different depths after the block. On average, there was more than 50\% signal increase in all depth ranges. In addition, the signal increased most within the $0.1 \mathrm{~mm}-0.4 \mathrm{~mm}$ depth range, where the capillary bed is located. According to the dual-wavelength measurement by AR-PAM, the average $\mathrm{sO}_{2}$ increased $\sim 4 \%$.

\section{Conclusion}

In this paper, peripheral blood vessels in two sites in patients' hands were imaged by PAM systems. From pre to post SGB block, there were a $50 \%$ increase in signal intensity of PAM and $4 \%$ increase in $\mathrm{sO}_{2}$, which agreed with the increased temperature and decreased pain level $[6,7]$. The results showed that blood perfusion increased after SBG, which is consistent with prior reports. Unlike other imaging methods, PAM can provide non-invasive high spatial resolution images as well as functional $\mathrm{sO}_{2}$ measurement. In these preliminary results, PAM showed its potential for helping both monitor and diagnose CRPS.

\section{Acknowledgments}

The authors would like to thank Prof. James Ballard for manuscript editing. This work was sponsored in part by National Institutes of Health grants DP1 EB016986 (NIH Director's Pioneer Award), R01 CA186567 (NIH Director's Transformative Research Award), and R01 CA159959. Maslov, Konstantin: Microphotoacoustics, Inc., which, however, did not support this work; Wang, Lihong: Microphotoacoustics, Inc. and Endra, Inc., which, however, did not support this work.

\section{References}

1. S. Bruehl, "An update on the pathophysiology of complex regional pain syndrome," Anesthesiology 113(3), 713-725 (2010).

2. T. Lubenow and M. Jaycox, "Current Trends in the Diagnosis and Treatment of Complex Regional Pain Syndrome," ASA Refresher Courses in Anesthesiology 40(1), 95-104 (2012).

3. R. J. Schwartzman, G. M. Alexander and J. Grothusen, "Pathophysiology of complex regional pain syndrome," Expert review of neurotherapeutics 6(5), 669-681 (2006).

4. L. Parkitny, J. H. McAuley, F. Di Pietro, T. R. Stanton, N. E. O'Connell, J. Marinus, J. J. van Hilten and G. L. Moseley, "Inflammation in complex regional pain syndrome A systematic review and meta-analysis," Neurology 80(1), 106-117 (2013).

5. F. van Eijs, J. Geurts, M. van Kleef, C. G. Faber, R. S. Perez, A. G. H. Kessels and J. Van Zundert, "Predictors of Pain Relieving Response to Sympathetic Blockade in Complex Regional Pain Syndrome Type 1," Anesthesiology 116(1), 113-121 (2012).

6. M. Schurmann, J. Zaspel, G. Gradl, A. Wipfel and F. Christ, "Assessment of the peripheral microcirculation using computer-assisted venous congestion plethysmography in post-traumatic complex regional pain syndrome type I," J Vasc Res 38(5), 453-461 (2001).

7. M. Dirckx, D. L. Stronks, G. Groeneweg and F. J. P. M. Huygen, "Effect of Immunomodulating Medications in Complex Regional Pain Syndrome A Systematic Review," Clin J Pain 28(4), 355-363 (2012).

8. X. Moisset and D. Bouhassira, "Brain imaging of neuropathic pain," Neuroimage 37(S80 (2007). 
9. D. Owen, Y. Bureau, A. Thomas, F. Prato and K. St Lawrence, "Quantification of pain-induced changes in cerebral blood flow by perfusion MRI," Pain-Journal of the International Association for the Study of Pain 136(1), 85-96 (2008).

10. A. Lebel, L. Becerra, D. Wallin, E. Moulton, S. Morris, G. Pendse, J. Jasciewicz, M. Stein, M. Aiello-Lammens and E. Grant, "fMRI reveals distinct CNS processing during symptomatic and recovered complex regional pain syndrome in children," Brain 131(7), 1854-1879 (2008).

11. J. A. Detre, J. S. Leigh, D. S. Williams and A. P. Koretsky, "Perfusion imaging," Magnetic Resonance in Medicine 23(1), 37-45 (2005).

12. R. C. Coghill, C. N. Sang, K. F. Berman, G. J. Bennett and M. J. Iadarola, "Global cerebral blood flow decreases during pain," Journal of Cerebral Blood Flow \& Metabolism 18(2), 141-147 (1998).

13. T. Okano, Y. Okuda, Y. Kimura, M. Mishio, M. Shinohara and T. Kitajima, "Use of near-infrared spectroscopy to evaluate stellate ganglion block," Region Anesth Pain M 26(2), 186-186 (2001).

14. M. Koban, S. Leis, S. Schultze-Mosgau and F. Birklein, "Tissue hypoxia in complex regional pain syndrome," Pain 104(1-2), 149-157 (2003).

15. Y. Zhao, Z. Chen, C. Saxer, S. Xiang, J. F. de Boer and J. S. Nelson, "Phase-resolved optical coherence tomography and optical Doppler tomography for imaging blood flow in human skin with fast scanning speed and high velocity sensitivity," Optics Letters 25(2), 114-116 (2000).

16. W. E. Ackerman, M. A. Munir and J.-M. Zhang, "Assessment of Laser Doppler Imaging for the Diagnosis of Complex Regional Pain Syndrome I," Journal of Neuropathic Pain \& Symptom Palliation 1(4), 13-20 (2005).

17. X. Wang, Y. Pang, G. Ku, X. Xie, G. Stoica and L. V. Wang, "Noninvasive laser-induced photoacoustic tomography for structural and functional in vivo imaging of the brain," Nat Biotechnol 21(7), 803-806 (2003).

18. H. F. Zhang, K. Maslov, G. Stoica and L. V. Wang, "Functional photoacoustic microscopy for high-resolution and noninvasive in vivo imaging," Nat Biotechnol 24(7), 848-851 (2006).

19. Y. Zhou, C. Zhang, D. K. Yao and L. H. V. Wang, "Photoacoustic microscopy of bilirubin in tissue phantoms," J. Biomed. Opt. 17(12), (2012).

20. C. Zhang, Y. Zhou, C. Y. Li and L. H. V. Wang, "Slow-sound photoacoustic microscopy," Appl Phys Lett 102(16), (2013).

21. J. Y. Liang, Y. Zhou, K. I. Maslov and L. H. V. Wang, "Cross-correlation-based transverse flow measurements using optical resolution photoacoustic microscopy with a digital micromirror device," J. Biomed. Opt. 18(9), (2013).

22. Y. Zhou, J. Y. Liang, K. I. Maslov and L. H. V. Wang, "Calibration-free in vivo transverse blood flowmetry based on cross correlation of slow time profiles from photoacoustic microscopy," Opt. Lett. 38(19), 3882-3885 (2013).

23. L. D. Liao, C. T. Lin, Y. Y. I. Shih, T. Q. Duong, H. Y. Lai, P. H. Wang, R. Wu, S. Tsang, J. Y. Chang and M. L. Li, "Transcranial imaging of functional cerebral hemodynamic changes in single blood vessels using in vivo photoacoustic microscopy," Journal of Cerebral Blood Flow \& Metabolism 32(6), 938-951 (2012).

24. J. Y. Liang, Y. Zhou, A. W. Winkler, L. D. Wang, K. I. Maslov, C. Y. Li and L. H. V. Wang, "Random-access optical-resolution photoacoustic microscopy using a digital micromirror device," Opt. Lett. 38(15), 2683-2686 (2013).

25. L. H. V. Wang and S. Hu, "Photoacoustic Tomography: In Vivo Imaging from Organelles to Organs," Science 335(6075), 1458-1462 (2012).

26. C. P. Favazza, L. A. Cornelius and L. V. Wang, "In vivo functional photoacoustic microscopy of cutaneous microvasculature in human skin," J Biomed Opt 16(2), 026004-026004-026005 (2011).

27. R. N. Harden, S. Bruehl, R. S. G. M. Perez, F. Birklein, J. Marinus, C. Maihofner, T. Lubenow, A. Buvanendran, S. Mackey, J. Graciosa, M. Mogilevski, C. Ramsden, M. Chont and J. J. Vatine, "Validation of proposed diagnostic criteria (the "Budapest Criteria") for Complex Regional Pain Syndrome," Pain 150(2), 268274 (2010).

28. K. Maslov, G. Stoica and L. V. Wang, "In vivo dark-field reflection-mode photoacoustic microscopy," Optics Letters 30(6), 625-627 (2005).

29. Y. Zhou, J. J. Yao and L. H. V. Wang, "Optical clearing-aided photoacoustic microscopy with enhanced resolution and imaging depth," Opt. Lett. 38(14), 2592-2595 (2013).

30. Y. Zhou, X. Yi, W. Xing, S. Hu, K. Maslov, and L. V. Wang, "Microcirculatory changes identified by photoacoustic microscopy in patients with complex regional pain syndrome type I after stellate ganglion blocks," J. Biomed. Opt. 19(8), 086017 (2014).

31. Y. Zhou, W. Xing, K. I. Maslov, Lynn A. Cornelius, and L. V. Wang, "Handheld photoacoustic microscopy to detect melanoma depth in vivo," Opt. Lett. 39(16), 4731-4734 (2014). 